\title{
Sensorineural Hearing Impairment and Subclinical Atherosclerosis in Rheumatoid Arthritis Patients Without Traditional Cardiovascular Risk Factors
}

\author{
Hector MACIAS-REYES, ${ }^{1}$ Sergio DURAN-BARRAGAN,,${ }^{2,3}$ Cynthia R. CARDENAS-CONTRERAS, ${ }^{1}$ \\ Cesar G. CHAVEZ-MARTIN, ${ }^{1}$ Eduardo GOMEZ-BAÑUELOS,${ }^{2}$ Rosa E. NAVARRO-HERNANDEZ, ${ }^{2}$ \\ Carlos O. YANOWSKY-GONZALEZ, ${ }^{2}$ Laura GONZALEZ-LOPEZ, ${ }^{5}$ \\ Jorge I. GAMEZ-NAVA, ${ }^{6}$ Monica VAZQUEZ-DEL MERCADO ${ }^{2,4}$ \\ ${ }^{1}$ Department of Otorhinolaryngology, Hospital Civil Fray Antonio Alcalde, Guadalajara, Mexico \\ ${ }^{2}$ Department of Rheumatology, Instituto De Investigación En Reumatología Y Del Sistema Musculoesquelético, Guadalajara, Mexico \\ ${ }^{3}$ Department of Rheumatology, Clínica De Investigación En Reumatología Y Obesidad, Guadalajara, Mexico \\ ${ }^{4}$ Department of Rheumatology, Hospital Civil Dr. Juan I. Menchaca, Guadalajara, Mexico \\ ${ }^{5}$ Department of Rheumatology, Hospital General Regional 110, IMSS, Guadalajara, Mexico \\ ${ }^{6}$ Unidad Médica De Alta Especialidad, CMNO, IMSS, Rheumatology, Guadalajara, Mexico
}

\begin{abstract}
Objectives: This study aims to evaluate the association of hearing impairment with carotid intima-media thickness and subclinical atherosclerosis in rheumatoid arthritis (RA) patients.

Patients and methods: A total of 41 RA patients ( 2 males, 39 females; mean age $46.5 \pm 10.2$ years; range 20 to 63 years) with no known traditional cardiovascular risk factors were included. Routine clinical and laboratory assessments for RA patients were performed. Pure tone air ( $250-8000 \mathrm{~Hz}$ ) and bone conduction $(250-6000 \mathrm{~Hz})$ thresholds were obtained, tympanograms and impedance audiometry were conducted. Sensorineural hearing impairment was defined if the average thresholds were $\geq 25$ decibels. Carotid intima-media thickness was assessed and classified with a cut-off point of $0.6 \mathrm{~mm}$.

Results: Thirteen patients (31.7\%) had normal audition, while 28 (68.3\%) had hearing impairment. Of these, 22 had bilateral sensorineural hearing impairment. Four patients had conductive hearing impairment (right in three patients and left in one patient). Patients with sensorineural hearing impairment had increased carotid intima-media thickness in the media segment of carotid common artery compared to patients with normal hearing (right ear $p=0.007$; left ear $p=0.075$ ). Thickening of the carotid intima-media thickness was associated with sensorineural hearing impairment in RA patients.

Conclusion: Rheumatoid arthritis patients should be evaluated by carotid intima-media thickness as a possible contributing factor of hearing impairment in patients without cardiovascular risk factors.

Keywords: Atherosclerosis; hearing loss; rheumatoid arthritis.
\end{abstract}

Hearing impairment (HI) is recognized as an important cause of disability strongly associated with older age, noise exposure, and low income., Diabetes mellitus, hypertension, and smoking are traditional cardiovascular risk factors considered as a contributing factor of $\mathrm{HI}$ among adults. ${ }^{2-6} \mathrm{HI}$ may be classified as conductive hearing impairment $(\mathrm{CHI})$, sensorineural hearing impairment (SNHI), and mixed.

Rheumatoid arthritis (RA) patients have higher frequency of $\mathrm{HI}$ than healthy controls (42\% vs. $15.9 \%){ }^{7} \mathrm{RA}$ is a disease characterized by 
inflammation of the synovial membrane of the diarthrodial joints, which include arthrodesis of the middle ear, (incudomalleolar and incudostapedial joints) causing $\mathrm{CHI}$ in up to $1.9 \%$ of the cases. ${ }^{4}$ Extra-articular involvement may also affect the auditory system targeting the inner ear, causing $\mathrm{SNHI}$ in $22 \%$ to $43 \%$ of the patients. ${ }^{4-10}$

Hearing impairment in RA is generally underdiagnosed; symptoms are progressive and patients are not aware of the damage until it interferes with their usual activities. ${ }^{5,6}$ Despite of the increasing number of research about this topic, there is no clear evidence indicating that $\mathrm{RA}$ per se might be a direct cause of $\mathrm{HI}$ since the physiopathology remains unclear., ${ }^{4,71-13}$ There is no consensus about methods to perform, when to screen for $\mathrm{HI}$ in RA patients with audiometric and clinical methods, or usefulness of these methods.

Recognized causes of $\mathrm{HI}$ related to RA are disease activity, vasculitis, neuritis, immune-complex mediated antibodies directed against epitopes, and ototoxicity caused by medications. ${ }^{14,15}$ The majority of the risk factors for SNHI reported in the literature are related to the cardiovascular system, as the inner ear may be vulnerable to circulatory alterations that can affect the labyrinthine artery.

Despite the probability that $\mathrm{HI}$ in RA patients may be associated with vascular damage, to our knowledge, previous research have not evaluated the association of cardiovascular damage with $\mathrm{HI}$ in RA patients. ${ }^{5,6}$ Therefore, in this study, we aimed to evaluate the association of hearing impairment with carotid intima-media thickness and subclinical atherosclerosis in RA patients.

\section{PATIENTS AND METHODS}

This cross sectional study included $41 \mathrm{RA}$ patients (2 males, 39 females; mean age 46.5 \pm 10.2 years; range 20 to 63 years), classified according to American College of Rheumatology criteria (1987). ${ }^{16}$ attending to the Rheumatology Service of Hospital Civil "Dr. Juan I. Menchaca", Guadalajara, Jalisco, Mexico, between August 2013 and December 2013. Subjects who were former or current smokers, subjects with a history of cardiovascular disease, hypertension, diabetes mellitus type 2, thyroid disease, renal impairment, malignancy, hepatic disease or hyperlipidemia, or who were previously treated with high doses of steroids (>10 mg/day prednisone or equivalent, including intravenous administration) were excluded.

A structured questionnaire was applied to all subjects to gather demographic information, medical history, date of diagnosis, and current treatment. Disease activity was evaluated using the disease activity score in 28 joints (DAS28) with C-reactive protein (CRP). ${ }^{17}$

Venous blood samples were drawn and allowed to clot at room temperature and subsequently centrifuged at 1500 relative centrifugal force (Rotanta 460R, Andreas Hettich GmbH \& Co. KG. Germany) for 10 minutes. The serum was stored at $-70{ }^{\circ} \mathrm{C}$ until all the samples were obtained and further analyses were performed. Erythrocyte sedimentation rate (ESR) was measured using the Wintrobe method. The CRP and rheumatoid factor (RF) were measured by standard techniques (RANDOX Laboratories Limited, 55 Diamond Road, Crumlin, Country Antrim, BT29 4QY, UK). Anti-cyclic citrullinated peptide (CCP) antibody $(\mathrm{U} / \mathrm{mL})$ was measured by second generation enzyme-linked immunosorbent assay (Axis-Shield Diagnostics Ltd. Scotland).

Pure tone air $(250-8000 \mathrm{~Hz})$ and bone conduction (250-6000 Hz) thresholds were obtained using the audiometer AA-97B (Rion Co. Ltd., Tokyo, Japan) calibrated according to Japanese Industrial Standard T1201-2000. SNHI was considered when the auditory threshold was $\geq 25 \mathrm{~dB}$ in more than one of the frequencies between 250 and $8000 \mathrm{~Hz}$ (inclusively) and when the difference between air and bone conductive thresholds was $<10 \mathrm{~dB}$. CHI was considered when air conductive threshold was $>20 \mathrm{~dB}$ and bone conductive $\leq 20 \mathrm{~dB}$.

Hearing loss disability was defined when there was a decibel sum hearing loss of $100 \mathrm{~dB}$ or greater at frequencies of 500,1000, 2000 and $3000 \mathrm{~Hz}$ in either ear, or of $50 \mathrm{~dB}$ or more in both ears at $4000 \mathrm{~Hz}$.

Impedance audiometry was measured using an IA-AZ-7 model tympanometer (Middelfart, Denmark) calibrated to International Organization for Standardization standards. Tympanograms were classified according to Jerger as $<$ A, As y C. 
Carotid intima-media thickness (cIMT) was assessed according to the recommendations defined by the Mannheim Carotid Intima-Media Thickness and Plaque Consensus (2004-20062011). ${ }^{18,19}$ by a single operator using a highresolution B-mode ultrasound (PHILIPS, Saronno, Italy) with a 9-MHz transducer. Two segments from the common carotid artery (CCA), one from the carotid bifurcation, and two from the internal carotid artery were evaluated. Mean cIMT values were calculated for each segment. Patients were classified according to the cIMT with a cut-off point of $0.6 \mathrm{~mm}$.

The study protocol was approved by the Institutional Review Board of "Hospital Civil Juan I. Menchaca", registered under the number 1068/10 and performed in accordance with the ethical standards laid down in the Declaration of Helsinki. A written informed consent was obtained from each patient.

\section{Statistical analysis}

Values are presented as mean \pm standard deviation and percentages as appropriate. Between-group differences were estimated by independent-sample Student's t test. Chi-square test (or Fisher's exact test) was used to compare categorical variables. Spearman's correlation coefficient was calculated for cIMT and air conduction velocity. All data were analyzed using PASW version 18.0 software (SPSS Inc., Chicago, IL, USA), considering a two-tailed level of $p<0.05$ statistically significant.

\section{RESULTS}

Mean disease duration was 7.05 (7.37) years. Twelve patients (29.2\%) had normal audition, while 29 (70.8\%) had HI; of these, 28 (68.3\%) had SHNI [22 (53.6\%) had bilateral SNHI: 24 (58.5\%) SNHI left and 26 (63.4\%) SNHI right], three patients $(7.3 \%)$ had right $\mathrm{CHI}$, and one patient (2.4\%) had left $\mathrm{CHI}$. None of the patients had hearing loss disability. RF and anti-CCP were positive in $20(48.5 \%)$ and $16(39.0 \%)$ patients, respectively. Mean DAS28-CRP was $3.19 \pm 1.28$ and DAS28-ESR was $4.10 \pm 1.26$. Disease activity by DAS28-CRP was classified as severe in four patients (79.7\%), moderate in 14 patients (34.1\%), low in 12 patients (29.3\%), and remission in 11 patients (26.8\%). Clinical and serological features are shown in Table 1.

Patients with SNHI were older than patients with normal audition $(49.68 \pm 8.8$ vs. $39.54 \pm 9.8$, $\mathrm{p}=0.002$ ). No statistical differences were found between normal audition and SNHI groups,

Table 1. Hearing impairment according to clinical and demographic characteristics in rheumatoid arthritis patients

\begin{tabular}{|c|c|c|c|c|c|c|c|}
\hline \multirow[b]{3}{*}{ All patients $(n=41)$} & \multicolumn{6}{|c|}{ Type of audition } & \multirow[b]{3}{*}{$p$} \\
\hline & \multicolumn{3}{|c|}{ Normal $(n=13)$} & \multicolumn{3}{|c|}{ SNHI $(n=28)$} & \\
\hline & $\mathrm{n}$ & $\%$ & Mean \pm SD & $\mathrm{n}$ & $\%$ & Mean \pm SD & \\
\hline Age (years) & & & $39.5 \pm 9.8$ & & & $49.7 \pm 8.8$ & 0.002 \\
\hline Female & 13 & 100 & & 26 & 92.9 & & 0.323 \\
\hline Disease duration (years) & & & $8.3 \pm 9.4$ & & & $6.5 \pm 6.3$ & 0.463 \\
\hline Rheumatoid factor (UI/L) & & & $159.6 \pm 184.3$ & & & $320.8 \pm 516.5$ & 0.385 \\
\hline Rheumatoid factor positive & 8 & 61.5 & & 12 & 42.8 & & 0.265 \\
\hline Anti-citrullinated cyclic peptide antibodies (UI/L) & & & $130.1 \pm 98.1$ & & & $308.0 \pm 358.9$ & 0.163 \\
\hline Anti-citrullinated cyclic peptide positive & 8 & 61.5 & & 8 & 28.5 & & 0.051 \\
\hline Erythrocyte sedimentation rate (mm/hour) & & & $22.0 \pm 12.6$ & & & $29.6 \pm 21.8$ & 0.256 \\
\hline C-reactive protein $(\mathrm{mg} / \mathrm{L})$ & & & $1.0 \pm 1.2$ & & & $5.1 \pm 18.3$ & 0.434 \\
\hline Disease activity index 28 joints-C-reactive protein & & & & & & & \\
\hline Remission & 3 & 23.1 & & 8 & 26.6 & & 0.437 \\
\hline Mild & 6 & 46.2 & & 6 & 21.4 & & \\
\hline Moderate & 3 & 23.1 & & 11 & 39.3 & & \\
\hline Severe & 1 & 7.7 & & 3 & 10.7 & & \\
\hline Treatment & & & & & & & \\
\hline Methotrexate & 14 & 82.4 & & 20 & 83.3 & & 0.626 \\
\hline Sulfasalazine & 9 & 52.9 & & 10 & 41.7 & & 0.537 \\
\hline Chloroquine & 5 & 29.4 & & 4 & 16.7 & & 0.450 \\
\hline Azathioprine & 0 & 0 & & 2 & 8.3 & & 0.502 \\
\hline Prednisone & 1 & 5.9 & & 2 & 8.3 & & 0.212 \\
\hline
\end{tabular}




\begin{tabular}{|c|c|c|c|c|c|c|c|}
\hline \multirow[t]{2}{*}{ Right carotid segments } & \multicolumn{7}{|c|}{ Air conduction velocity $(\mathrm{Hz})$} \\
\hline & 250 & 500 & 1000 & 2000 & 4000 & 6000 & 8000 \\
\hline \multicolumn{8}{|l|}{ Proximal CCA } \\
\hline $\mathrm{cc}$ & -0.111 & -0.067 & -0.123 & -0.042 & -0.021 & 0.062 & 0.101 \\
\hline$p$ & 0.490 & 0.678 & 0.445 & 0.794 & 0.898 & 0.699 & 0.530 \\
\hline \multicolumn{8}{|l|}{ Media CCA } \\
\hline $\mathrm{cc}$ & 0.102 & 0.247 & 0.083 & 0.157 & 0.218 & 0.241 & 0.188 \\
\hline$p$ & 0.524 & 0.120 & 0.607 & 0.328 & 0.172 & 0.129 & 0.240 \\
\hline \multicolumn{8}{|l|}{ Distal CCA } \\
\hline $\mathrm{cc}$ & 0.078 & 0.120 & 0.039 & 0.246 & $0.370^{*}$ & $0.358^{*}$ & $0.350^{*}$ \\
\hline$p$ & 0.628 & 0.453 & 0.807 & 0.120 & 0.017 & 0.022 & 0.025 \\
\hline \multicolumn{8}{|l|}{ Bulb } \\
\hline $\mathrm{cc}$ & 0.068 & 0.144 & 0.022 & 0.063 & 0.092 & 0.107 & 0.056 \\
\hline$p$ & 0.671 & 0.369 & 0.890 & 0.694 & 0.568 & 0.507 & 0.728 \\
\hline \multicolumn{8}{|l|}{ Proximal ICA } \\
\hline $\mathrm{cc}$ & -0.041 & 0.030 & -0.039 & 0.050 & 0.025 & 0.040 & 0.013 \\
\hline$p$ & 0.797 & 0.853 & 0.807 & 0.755 & 0.878 & 0.804 & 0.935 \\
\hline \multicolumn{8}{|l|}{ Distal ICA } \\
\hline $\mathrm{cc}$ & 0.133 & 0.097 & 0.024 & 0.062 & 0.204 & 0.109 & -0.016 \\
\hline$p$ & 0.406 & 0.545 & 0.881 & 0.702 & 0.200 & 0.496 & 0.920 \\
\hline
\end{tabular}

although higher titles of RF, anti-CCP, ESR and $\mathrm{CRP}$ were detected in the SHNI group. No statistical significant relationship was found between positivity for RF and anti-CCP between SNHI and normal hearing RA groups (data not shown).

Rheumatoid arthritis patients with right SNHI had thicker right media CCA compared to RA patients with normal audition $(0.21 \pm 0.29$ vs. $0.02 \pm 0.10, p=0.007$ ); patients with left SHNI did not have difference in left media CCA compared to patients with normal audition $(0.20 \pm 0.30$ vs. $0.06 \pm 0.18, p=0.075)$. Other segments such as proximal and distal CCA, bulb, proximal and distal internal carotid artery in both sides did not show any significant differences according to right and left SHNI groups.

We detected a statistically significantly positive correlation between right distal CCA cIMT values

Table 3. Left carotid segments correlation with air conduction velocity in left ear

\begin{tabular}{|c|c|c|c|c|c|c|c|}
\hline \multirow[t]{2}{*}{ Left carotid segments } & \multicolumn{7}{|c|}{ Air conduction velocity $(\mathrm{Hz})$} \\
\hline & 250 & 500 & 1000 & 2000 & 4000 & 6000 & 8000 \\
\hline \multicolumn{8}{|l|}{ Proximal CCA } \\
\hline $\mathrm{cc}$ & 0.045 & 0.096 & -0.079 & 0.167 & 0.148 & 0.279 & 0.231 \\
\hline$p$ & 0.778 & 0.549 & 0.625 & 0.295 & 0.355 & 0.077 & 0.147 \\
\hline \multicolumn{8}{|l|}{ Media CCA } \\
\hline $\mathrm{cc}$ & 0.064 & 0.233 & 0.156 & 0.257 & 0.298 & $0.354^{*}$ & $0.325^{*}$ \\
\hline$p$ & 0.691 & 0.143 & 0.330 & 0.105 & 0.059 & 0.023 & 0.038 \\
\hline \multicolumn{8}{|l|}{ Distal CCA } \\
\hline cc & 0.132 & 0.170 & 0.014 & 0.232 & 0.195 & 0.249 & 0.268 \\
\hline$p$ & 0.411 & 0.287 & 0.929 & 0.145 & 0.221 & 0.116 & 0.090 \\
\hline \multicolumn{8}{|l|}{ Bulb } \\
\hline $\mathrm{cc}$ & -0.132 & -0.060 & -0.177 & 0.057 & -0.073 & 0.004 & 0.004 \\
\hline$p$ & 0.412 & 0.711 & 0.267 & 0.721 & 0.652 & 0.983 & 0.981 \\
\hline \multicolumn{8}{|l|}{ Proximal ICA } \\
\hline $\mathrm{cc}$ & -0.047 & -0.048 & -0.224 & -0.099 & -0.107 & -0.056 & -0.055 \\
\hline$p$ & 0.771 & 0.764 & 0.159 & 0.536 & 0.504 & 0.726 & 0.732 \\
\hline \multicolumn{8}{|l|}{ Distal ICA } \\
\hline $\mathrm{cc}$ & 0.128 & 0.166 & -0.110 & 0.033 & 0.002 & 0.063 & 0.013 \\
\hline$p$ & 0.425 & 0.301 & 0.495 & 0.835 & 0.988 & 0.695 & 0.938 \\
\hline
\end{tabular}


and abnormal audition at $4000 \mathrm{~Hz}$ [correlation coefficient (cc) 0.370, p=0.017), $6000 \mathrm{~Hz}$ (cc 0.358, p=0.022) and $8000 \mathrm{~Hz}$ (cc 0.350, $\mathrm{p}=0.025)]$ as shown in Table 2.

In addition, we found a positive correlation between medial CCA cIMT values and abnormal audition at $6000 \mathrm{~Hz}$ (cc 0.354, p=0.023) and $8000 \mathrm{~Hz}$ (cc 0.325, p=0.038) in the contralateral ear and artery, as shown in Table 3.

\section{DISCUSSION}

The pathogenesis of $\mathrm{HI}$ in $\mathrm{RA}$ has been a matter of debate for decades, and today, it is still poorly understood. Causes of $\mathrm{HI}$ have been described as multifactorial in the previous studies. SNHI seems to be the hearing damage most frequently found in RA patients, and it has been attributed to disease activity, older age and extra-articular involvement such as vasculitis. ${ }^{8}$ In addition, majority of drugs used in the treatment of RA such as non-steroidal anti-inflammatory drugs and disease-modifying antirheumatic drugs, given to patients with more active disease, have also been recognized as potential contributors to SNHI. ${ }^{15,20-22}$ Thus, an association between disease activity and $\mathrm{HI}$ has not been not consistent.

Pascual-Ramos et al. $^{5}$ in a previous report demonstrated that $24 \%$ of 113 RA patients had HI. In a follow-up of their patients years later, they found in the adjusted Cox proportional model that cumulative DAS28-CRP was the only variable to predict incidental $\mathrm{HI}$ (odds ratio: 1.8, 95\%; confidence interval: 1.1-2.7; $p=0.01$ ). Almost 13\% of RA patients with short disease duration and low disease activity developed incidental $\mathrm{HI}$ within one year. ${ }^{6}$ In 2005, Takatsu et al. ${ }^{7}$ reported the frequency of SNHI in RA patients to be higher than normal controls (36.1\% vs. $13.9 \%$ ) and associated SNHI to ESR $(p<0.05)$, plasma interleukin-6 $(p<0.05)$, and plasma matrix metalloproteinase-3 $(p<0.001)$ suggesting that systemic inflammatory process might trigger $\mathrm{HI}$. On the other hand, Murdin et al. ${ }^{4}$ did not find any relationship between hearing thresholds and markers of disease activity or other rheumatological parameters.

Cardiovascular disease in RA patients reduces life expectancy between six and seven years, ${ }^{23}$ due to myocardial infarction, stroke, hypertension, and subclinical atherosclerosis. ${ }^{19}$ In the last decade, RA has been recognized as a cardiovascular risk factor per se. ${ }^{13}$ We have previously reported that cIMT correlates with levels of anti-CCP antibodies $(r=0.513, p=0.001)$, CRP $(r=0.799, p<0.001)$, tumor necrosis factor alpha $(r=0.642, p=0.001)$, and interleukin-6 $(\mathrm{r}=0.751, \mathrm{p}<0.001)$ in $\mathrm{RA}$ patients. In multiple regression analysis, cIMT was independently associated with CRP $(\mathrm{p}<0.001)$ and anti-CCP antibodies ( $p=0.03) .{ }^{19}$ Gökmen et al. ${ }^{24}$ have found that cIMT was positively correlated with the number of metabolic syndrome risk components in RA patients. They have shown positive correlations between cIMT and age, ESR, $\mathrm{CRP}$, and systolic and diastolic blood pressures. On the other hand, cIMT has been associated with homocysteine levels in RA patients treated with methotrexate. ${ }^{25}$ This increased risk is not exclusive to RA patients, increased cIMT has been reported in female patients with fibromyalgia. ${ }^{26}$

Hearing impairment may be associated with vascular damage in RA patients. Subclinical atherosclerosis may reduce the normal blood flow of brain circulation and this reduction may compromise the inner ear cells which are more sensitive to hypoxemic risk, leading to changes in the perception of high frequency sounds. Another possible explanation is that thickening of the intima-media carotid arteries may affect the inner ear blood flow, especially the labyrinthine artery, leading to SNHI by the same mechanism mainly at high frequencies. To our knowledge, we have shown for the first time that RA patients with SNHI had increased intima-media thickness in the carotid arteries. In a study of 381 consecutive patients who underwent coronary angiography for symptoms suggesting ischemic heart disease, Erkan et al. ${ }^{27}$ found that the angiographic severity and extent of coronary artery disease are significantly and independently correlated with the degree of hearing loss. There was a statistically significant positive correlation between the degree of hearing loss at all frequencies analyzed (250, 500, 1,000, 2,000, 4,000 Hz) and the Gensini score ( $<<0.05$ for all frequencies). Furthermore, in a case control study, Ciccone et al. $^{28}$ evaluated 29 patients with idiopathic sudden sensorineural hearing loss and 29 controls and showed that the two groups did not differ with regards to cIMT and other cardiovascular risk factors; however, flow-mediated dilation of 
the brachial artery predisposed individuals to develop idiopathic sudden sensorineural hearing loss ( $p=0.03$, odds ratio: 1.4 ).

There is evidence to suggest that hearing and vestibular function might be influenced by disease activity and autoimmune processes. RA patients may develop autoimmune inner-ear disease due to SNHI. There are some reports indicating that anti-tumor necrosis factor alpha agents (etanercept, adalimumab and infliximab) may offer additional treatment options for patients with autoimmune hearing loss. ${ }^{20-22}$

Hearing impairment in RA is a subclinical comorbid state. Since most of the patients are not aware of their $\mathrm{HI}$ and have no clinical complaints, it is important that physicians consider hearing damage in these patients. ${ }^{2,3,14}$

Despite of the increasing number of research on this topic, there is no clear evidence for the direct cause of $\mathrm{HI}$, since the physiopathology remains unclear. ${ }^{2,6,11}$ There is no consensus about audiometric evaluation methods and clinical screening in RA patients tested for $\mathrm{HI}$. In this context, we are unable to differentiate the importance of RA itself and other comorbidities causing $\mathrm{HI}$.

Our study has some limitations. Due to the cross sectional methodology of the study, we were unable to discriminate the impact of the variables across time in SNHI. And because of the small sample size, we might have missed a positive association of clinical and laboratorial variables with SNHI.

In conclusion, to our knowledge, we reported for the first time a positive association of subclinical atherosclerosis with SNHI in RA patients. As we have shown, auditory symptoms are common features associated with the disease. Follow-up appointments should be regarded as opportunities to enquire about hearing loss. Referral for screening and early treatment or rehabilitation increases the likelihood of a successful outcome. Further studies are required to elucidate the principal mechanism of SNHI, measure the labyrinthine artery by magnetic resonance imaging or computed angiotomography, and address cardiovascular risk factors and their impact in $\mathrm{HI}$ in RA patients.

\section{Declaration of conflicting interests}

The authors declared no conflicts of interest with respect to the authorship and/or publication of this article.

\section{Funding}

The authors received no financial support for the research and/or authorship of this article.

\section{REFERENCES}

1. Agrawal Y, Platz EA, Niparko JK. Prevalence of hearing loss and differences by demographic characteristics among US adults: data from the National Health and Nutrition Examination Survey, 1999-2004. Arch Intern Med 2008;168:1522-30.

2. Mohammadi S, Labbafinejad Y, Attarchi M. Combined effects of ototoxic solvents and noise on hearing in automobile plant workers in Iran. Arh Hig Rada Toksikol 2010;61:267-74.

3. Mohammadi S, Mazhari MM, Mehrparvar AH, Attarchi MS. Effect of simultaneous exposure to occupational noise and cigarette smoke on binaural hearing impairment. Noise Health 2010;12:187-90.

4. Murdin L, Patel S, Walmsley J, Yeoh LH. Hearing difficulties are common in patients with rheumatoid arthritis. Clin Rheumatol 2008;27:637-40.

5. Pascual-Ramos V, Contreras-Yáñez I, Enríquez L, Valdés S, Ramírez-Anguiano J. Hearing impairment in a tertiary-care-level population of Mexican rheumatoid arthritis patients. J Clin Rheumatol 2012;18:393-8.

6. Pascual-Ramos V, Contreras-Yáñez I, Rivera-Hoyos $\mathrm{P}$, Enríquez L, Ramírez-Anguiano J. Cumulative disease activity predicts incidental hearing impairment in patients with rheumatoid arthritis (RA). Clin Rheumatol 2014;33:315-21.

7. Takatsu M, Higaki M, Kinoshita H, Mizushima Y, Koizuka I. Ear involvement in patients with rheumatoid arthritis. Otol Neurotol 2005;26:755-61.

8. García Callejo FJ, Conill Tobías N, Muñoz Fernández N, de Paula Vernetta C, Alonso Castañeira I, Marco Algarra J. Hearing impairment in patients with rheumatoid arthritis. Acta Otorrinolaringol Esp 2007;58:232-8.

9. Kakani RS, Mehra YN, Deodhar SD, Mann SB, Mehta S. Audiovestibular functions in rheumatoid arthritis. J Otolaryngol 1990;19:100-2.

10. Ozcan M, Karakuş MF, Gündüz $\mathrm{OH}$, Tuncel U, Sahin H. Hearing loss and middle ear involvement in rheumatoid arthritis. Rheumatol Int 2002;22:16-9.

11. Poorey VK, Khatri R. Study of Auditory function in Rheumatoid Arthritis. Indian $\mathrm{J}$ Otolaryngol Head Neck Surg 2001;53:261-3.

12. Eckert MA, Cute SL, Vaden KI Jr, Kuchinsky SE, Dubno JR. Auditory cortex signs of age-related hearing loss. J Assoc Res Otolaryngol 2012;13:703-13. 
13. Peters MJ, Symmons DP, McCarey D, Dijkmans BA, Nicola P, Kvien TK, et al. EULAR evidencebased recommendations for cardiovascular risk management in patients with rheumatoid arthritis and other forms of inflammatory arthritis. Ann Rheum Dis 2010;69:325-31.

14. Silan F, Demirci L, Egeli A, Egeli E, Onder HI, Ozturk $\mathrm{O}$, et al. Syndromic etiology in children at schools for the deaf in Turkey. Int J Pediatr Otorhinolaryngol 2004;68:1399-406.

15. Stevens G, Flaxman S, Brunskill E, Mascarenhas M, Mathers CD, Finucane M. Global and regional hearing impairment prevalence: an analysis of 42 studies in 29 countries. Eur J Public Health 2013;23:146-52.

16. Arnett FC, Edworthy SM, Bloch DA, McShane DJ, Fries JF, Cooper NS, et al. The American Rheumatism Association 1987 revised criteria for the classification of rheumatoid arthritis. Arthritis Rheum 1988;31:315-24.

17. Prevoo ML, van 't Hof MA, Kuper $\mathrm{HH}$, van Leeuwen MA, van de Putte LB, van Riel PL. Modified disease activity scores that include twenty-eight-joint counts. Development and validation in a prospective longitudinal study of patients with rheumatoid arthritis. Arthritis Rheum 1995;38:44-8.

18. Touboul PJ, Hennerici MG, Meairs S, Adams H, Amarenco P, Bornstein N, et al. Mannheim carotid intima-media thickness and plaque consensus (20042006-2011). An update on behalf of the advisory board of the 3rd, 4th and 5th watching the risk symposia, at the 13th, 15th and 20th European Stroke Conferences, Mannheim, Germany, 2004, Brussels, Belgium, 2006, and Hamburg, Germany, 2011. Cerebrovasc Dis 2012;34:290-6.

19. Vázquez-Del Mercado M, Nuñez-Atahualpa L, Figueroa-Sánchez M, Gómez-Bañuelos E, RochaMuñoz AD, Martín-Márquez BT, et al. Serum levels of anticyclic citrullinated peptide antibodies, interleukin-6, tumor necrosis factor- $\alpha$, and C-reactive protein are associated with increased carotid intima-media thickness: a cross-sectional analysis of a cohort of rheumatoid arthritis patients without cardiovascular risk factors. Biomed Res Int
2015;2015:342649.

20. Gazeau P, Saraux A Devauchelle-Pensec V, Cornec D. Long-term efficacy of infliximab in autoimmune sensorineural hearing loss associated with rheumatoid arthritis. Rheumatology (Oxford) 2014;53:1715-6.

21. Morovic Vergles J, Radic M, Kovacic J, Salamon L. Successful use of adalimumab for treating rheumatoid arthritis with autoimmune sensorineural hearing loss: two birds with one stone. J Rheumatol 2010;37:1080-1.

22. Street I, Jobanputra P, Proops DW. Etanercept, a tumour necrosis factor alpha receptor antagonist, and methotrexate in acute sensorineural hearing loss. $\mathrm{J}$ Laryngol Otol 2006;120:1064-6.

23. Lassere MN, Rappo J, Portek IJ, Sturgess A, Edmonds JP. How many life years are lost in patients with rheumatoid arthritis? Secular cause-specific and allcause mortality in rheumatoid arthritis, and their predictors in a long-term Australian cohort study. Intern Med J 2013;43:66-72.

24. Gokmen F, Temiz A, Akbal A, Sen H, Zateri C, Gokmen E, et al. Number of metabolic syndrome risk factors is related to carotid intima-media thickness in rheumatoid arthritis patients. Arch Rheumatol 2015;30:124-9.

25. Hayta E, Hizmetli S, Atalar MH, Çinar Z. Association of plasma homocysteine level and carotid intima-media thickness in rheumatoid arthritis patients receiving methotrexate. Arch Rheumatol 2015;30:214-20.

26. Boluk H, Ozturk GT, Cotmert D, Ersoz M. Increased carotid intima-media thickness in female patients with fibromyalgia: a preliminary study. Arch Rheumatol 2015;30:307-10.

27. Erkan AF Beriat GK, Ekici B, Doğan C, Kocatürk S, Töre HF. Link between angiographic extent and severity of coronary artery disease and degree of sensorineural hearing loss. Herz 2015;40:481-6.

28. Ciccone MM, Cortese F, Pinto M, Di Teo C, Fornarelli F, Gesualdo M, et al. Endothelial function and cardiovascular risk in patients with idiopathic sudden sensorineural hearing loss. Atherosclerosis 2012;225:511-6. 